ARTICLE

Received 30 Jan 2015 | Accepted 5 Jun 2015 | Published 20 Jul 2015 DOl: 10.1038/ncomms8747 OPEN

\title{
Non-wetting surface-driven high-aspect-ratio crystalline grain growth for efficient hybrid perovskite solar cells
}

Cheng $\mathrm{Bi}^{1,2}$, Qi Wang ${ }^{1,2}$, Yuchuan Shao ${ }^{1,2}$, Yongbo Yuan ${ }^{1,2}$, Zhengguo Xiao ${ }^{1,2}$ \& Jinsong Huang ${ }^{1,2}$

Large-aspect-ratio grains are needed in polycrystalline thin-film solar cells for reduced charge recombination at grain boundaries; however, the grain size in organolead trihalide perovskite (OTP) films is generally limited by the film thickness. Here we report the growth of OTP grains with high average aspect ratio of 2.3-7.9 on a wide range of non-wetting hole transport layers (HTLs), which increase nucleus spacing by suppressing heterogeneous nucleation and facilitate grain boundary migration in grain growth by imposing less drag force. The reduced grain boundary area and improved crystallinity dramatically reduce the charge recombination in OTP thin films to the level in OTP single crystals. Combining the high work function of several HTLs, a high stabilized device efficiency of $18.3 \%$ in low-temperature-processed planar-heterojunction OTP devices under 1 sun illumination is achieved. This simple method in enhancing OTP morphology paves the way for its application in other optoelectronic devices for enhanced performance.

\footnotetext{
${ }^{1}$ Department of Mechanical and Materials Engineering, College of Engineering, University of Nebraska-Lincoln, Lincoln, Nebraska 68588-0656, USA.

${ }^{2}$ Nebraska Center for Materials and Nanoscience, University of Nebraska-Lincoln, Lincoln, Nebraska 68588-0656, USA. Correspondence and requests for materials should be addressed to J.H. (email: jhuang2@unl.edu).
} 
rganolead triiodide perovskite (OTP)-based solar cells have been attracting increasing attention from the solar energy community in the past few years due to the rapid increase of device power conversion efficiency (PCE) to the level of silicon solar cells and low-cost prospectus of this technology in terms of raw materials and device fabrication ${ }^{1-11}$. Nearly every important progress in device efficiency enhancement counts on the improved material quality of the OTP films for both mesoporous structure and planar heterojunction $(\mathrm{PHJ})$ structure OTP devices ${ }^{1-3,12-17}$. Similar to any polycrystalline thin film solar cells, larger OTP grains with less grain boundaries have been shown to increase the efficiency of OTP devices ${ }^{3,12}$. More evidences have shown that grain boundaries in OTP films might cause increasing charge recombination due to the presence of large density of charge traps $4,13,14,18-20$. Our recent studies have shown that the carrier diffusion length can be boosted to record value of above $200 \mu \mathrm{m}$ under 1 sun illumination and larger than $3 \mathrm{~mm}$ under weak light at room temperature for both electrons and holes in OTP single crystals that have no grain boundaries ${ }^{14}$. Although it is plausible to develop record high efficiency device with the OTP single crystals, the increasing material cost and lacking of techniques to scale up OTP single-crystal growth disgrace its prospectus for large-area, low-cost solar panels. An ideal OTP solar cell should have thin OTP layers with thickness sufficient to absorb most of the sun light, which is around $300-600 \mathrm{~nm}$ thanks to the very high extinction efficiency of OTP materials ${ }^{6}$, and with grain size as large as the cells to simulate the single-crystal solar cells. However, the size of the OTP grains grown by many methods is generally limited by the film thickness of the OTP films $3,12,21$.

A trick to grow large-size clear single-crystal ices is to use clean, smooth and non-wetting plastic container to prevent the formation of too dense nuclei from heterogeneous nucleation. In this manuscript, we used the same trick to grow OTP films with large crystalline grains on a wide range of non-wetting hole transport layers (HTLs). The average grain size/thickness aspect ratio in the OTP films grown on non-wetting HTLs reaches $2.3-7.9$, which dramatically reduces charge trap density by $10-100$-fold and boosts the PCE of OTP planar heterojunction solar cells to $18.3 \%$.

\section{Results}

Mechanism of OTP grain growth on non-wetting surfaces. The mechanism of growing large-size OTP grain on non-wetting surfaces is illustrated in Fig. 1, which shows the difference of nucleation and grain growth process on a wetting and a nonwetting surface. Here the two-step thermal annealing-assisted interdiffusion method was employed that forms continuous and pinhole-free OTP films ${ }^{12}$. The first step of OTP film formation is OTP nucleation on the substrates after the chemical reaction of $\mathrm{PbI}_{2}$ and MAI (MA is methylammonium). Then the followed drying and thermal annealing processes drive the interdiffusion and complete reaction of the two precursors, which forms and grows the OTP grains. It has been shown that thermal annealing increases the OTP grain size to above $300 \mathrm{~nm}$ on the wetting surface of poly(3,4-ethylenedioxythiophene) polystyrene sulfonate (PEDOT:PSS), after $2 \mathrm{~h}$ annealing at $105^{\circ} \mathrm{C}$, while further increasing of thermal annealing time causes the decomposition of OTP films without significantly increasing the grain size. It indicates the grain boundaries were pinned, most likely, by the impurities lying in the grain boundaries ${ }^{22}$. Adding solvent vapour during thermal annealing can de-pin the grain boundary and drive larger grain formation, however, the average grain size in OTP films grown on PEDOT:PSS is still limited to be the film thickness ${ }^{12}$. Another feature of the solvent-annealed OTP

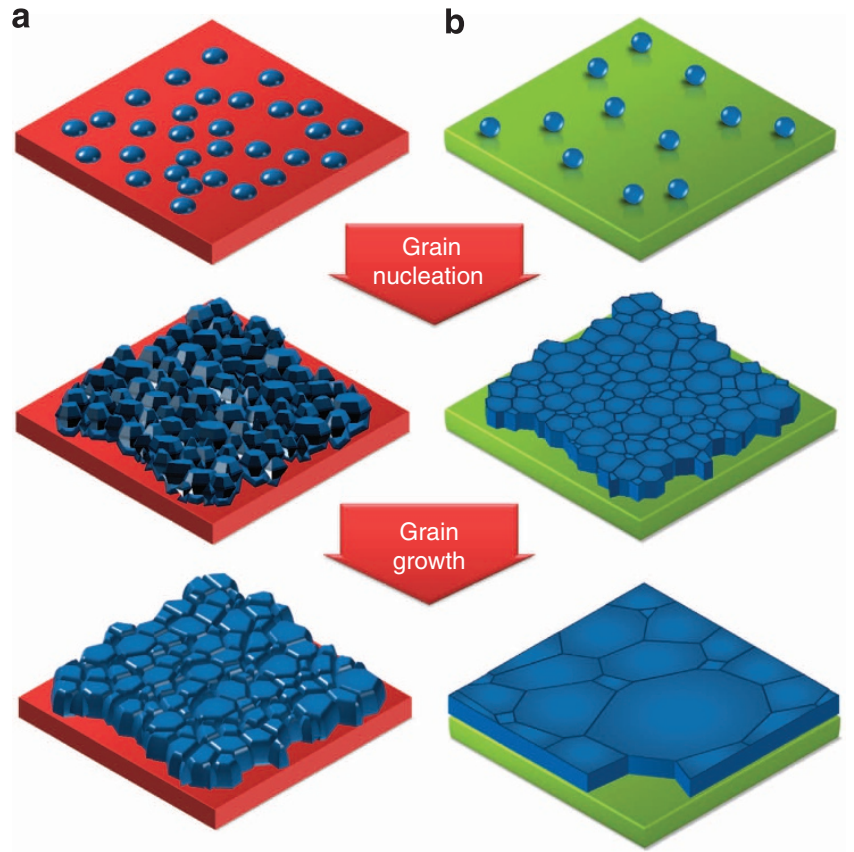

Figure 1 | Mechanism of large grain growth on non-wetting HTLs. Illustration of the nucleation and growth of the grains on wetting (a) and non-wetting HTLs (b) after thermal annealing.

films is the presence of some small grains close to PEDOT:PSS side and tilted grain boundaries between the large grains, as shown in cross-section scanning electron microscopy (SEM) image in Supplementary Fig. 1. This can be explained by the surface tension dragging force from the wetting PEDOT:PSS substrates, which reduces the grain boundary mobility. Such dragging force dramatically diminishes if the substrate is nonwetting and smooth, yielding a higher grain boundary mobility, which enables the growth of larger grains. The very smooth surface of the polymer HTL also suppresses the nucleation in small cavity and contributes to the high grain boundary mobility.

Large aspect ratio OTP grain growth on non-wetting HTLs. The proposed grain growth mechanism was demonstrated by studying the grain morphology of methylammonium lead triiodide $\left(\mathrm{MAPbI}_{3}\right)$ films on a wide range of wetting and non-wetting polymer substrates, including polyvinyl alcohol (PVA), PEDOT: PSS, crosslinked N4,N4'-bis(4-(6-((3-ethyloxetan-3-yl)methoxy) hexyl)phenyl)-N4,N4'-diphenylbiphenyl-4,4'-diamine (c-OTPD), poly(bis(4-phenyl)(2,4,6-trimethylphenyl)amine) (PTAA) and poly ( $N$-9'-heptadecanyl-2,7-carbazole-alt-5,5-( $4^{\prime}, 7^{\prime}$-di-2-thienyl- $2^{\prime}, 1^{\prime}, 3^{\prime}$ benzothiadiazole)) (PCDTBT). The chemical structures of these polymers are shown in Supplementary Fig. 2. The hydroxyl group in PVA and PEDOT:PSS provides wetting surfaces, while other polymers are hydrophobic. The presence of oxygen group in c-OTPD makes it less hydrophobic as PTAA or PCDTBT. The contacting angles of water on these polymers shown in Fig. 2a are $10^{\circ}, 12^{\circ}, 79^{\circ}, 105^{\circ}$ and $108^{\circ}$ for PVA, PEDOT:PSS, c-OTPD, PTAA and PCDTBT, respectively. The measured contact angle measured here represents the wetting capability of the different substrate surfaces to water, and we hypothesize it is also an indication of the wetting capability of these surfaces to solid-state OTP because of its hydrophilic property, as it can be dissolved in many polar solvents like $N, N$-dimethylformamide (DMF). Solution process was used to fabricate the HTLs and the $\mathrm{MAPbI}_{3}$ layer, where the HTL was first spun, and then some were crosslinked by ultraviolet curing and 
a b

C
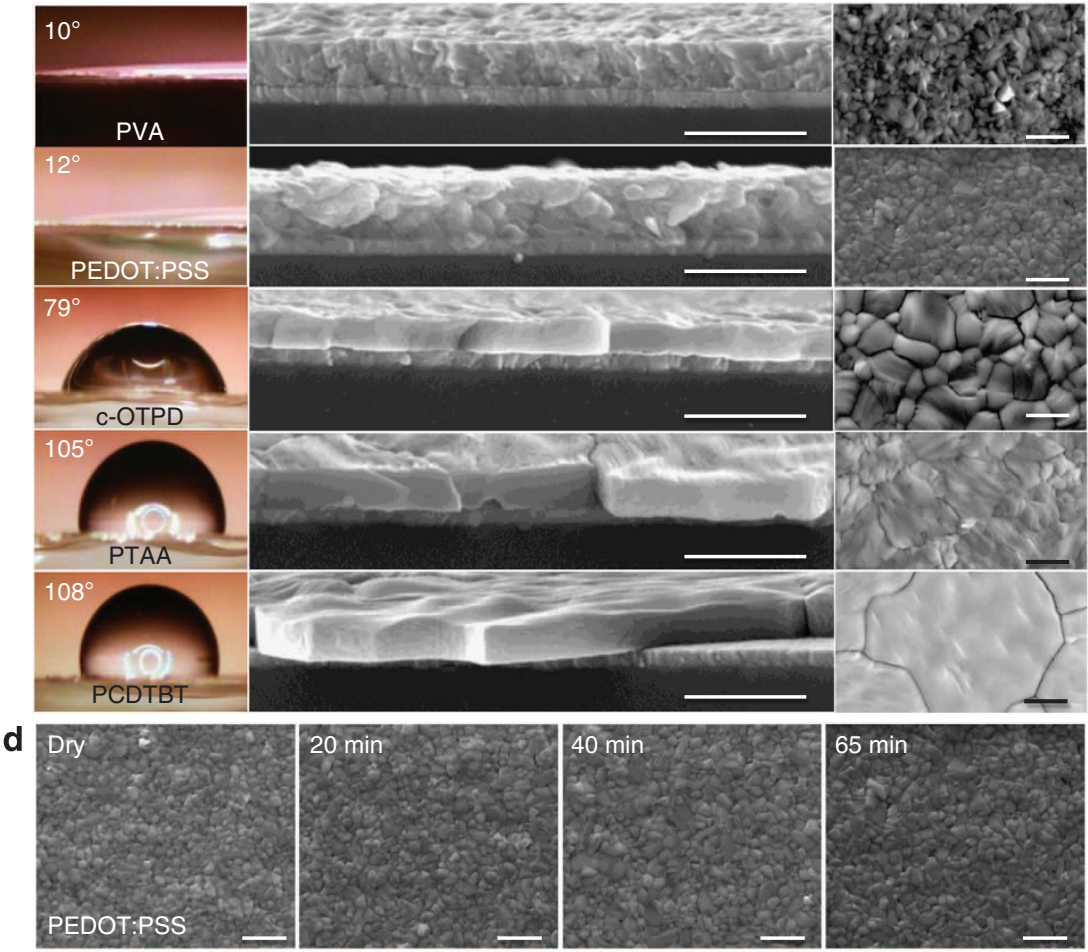

e
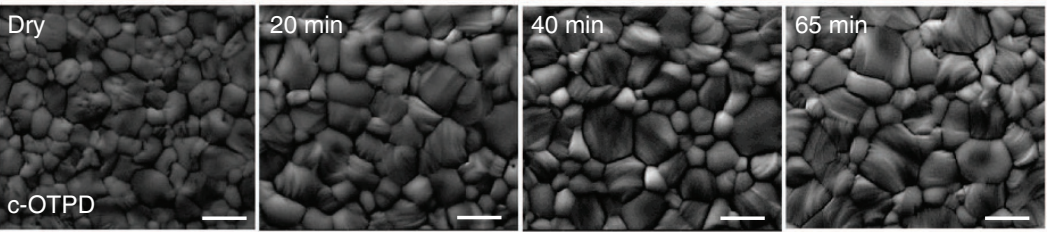

f
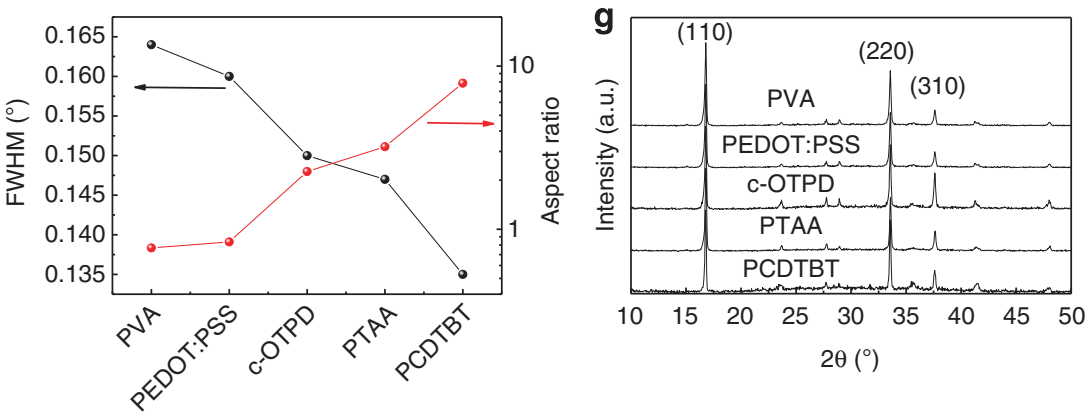

Figure 2 | Morphology of MAPbl $_{3}$ films grown on wetting and non-wetting HTLs. The contact angle of water on the varied HTLs (a), the crosssection SEM (b), top-view SEM (c) and X-ray diffraction patterns of the 360-nm MAPbl ${ }_{3}$ grown on PVA-, PEDOT:PSS-, c-OTPD-, PTAA- and PCDTBTcovered ITO substrates $(\mathbf{g})$. Scale bars, $1 \mu \mathrm{m}$ in $\mathbf{b}, \mathbf{c} ;(\mathbf{d}, \mathbf{e})$ the top-view SEM images of the MAPbl ${ }_{3}$ grown on PEDOT:PSS (top row) and c-OTPD (bottom row) right after drying and after 20,40 and $65 \mathrm{~min}$ of thermal annealing at $105^{\circ} \mathrm{C}$. Scale bar, $1 \mu \mathrm{m}$; (f) $\mathrm{HTL}$-dependent X-ray diffraction (110) peak full width at half maximum (FWHM) and average grain size/thickness aspect ratio of the $\mathrm{MAPbl}_{3}$.

thermal annealing. The fabrication details of the HTLs are shown in the Methods section. The $\mathrm{MAPbI}_{3}$ film was fabricated by thermal annealing-induced interdiffusion method ${ }^{5,10,12}$. The hydrophobic surfaces of non-wetting HTLs imposed a challenge in fabricating continuous pinhole-free hydrophilic OTP films on them although it is good for the large grain growth. Formation of continuous $\mathrm{PbI}_{2}$ films on the HTLs is a prerequisite for the continuous OTP film formation. To form continuous $\mathrm{PbI}_{2}$ on the HTLs, the $\mathrm{PbI}_{2}$ solution was heated to $110^{\circ} \mathrm{C}$ and quickly dripped on the HTLs for spin coating, which yielded smooth $\mathrm{PbI}_{2}$ films over the whole substrate as shown by the optical microscope images in Supplementary Fig. 3a. At lower solution temperature, $\mathrm{PbI}_{2}$ solution formed nonuniform films with many spots, as shown in Supplementary Fig. 3 b.
Here all the $\mathrm{MAPbI}_{3}$ films were thermal annealed for $65 \mathrm{~min}$ at $105^{\circ} \mathrm{C}$. The cross-section and top-view SEM images of the $\mathrm{MAPbI}_{3}$ films on these HTLs shown in Fig. 2b,c reveal a clear correlation between the substrate surface wetting capability and the grain morphology: First, the average grain size is much larger for $\mathrm{MAPbI}_{3}$ films on hydrophobic HTLs. The grain size distribution for $\mathrm{MAPbI}_{3}$ on various HTLs was summarized in Supplementary Fig. 4. The sizes of $\mathrm{MAPbI}_{3}$ grains on wetting PVA $(277 \mathrm{~nm})$ and PEDOT:PSS $(301 \mathrm{~nm})$ are smaller than the film thickness $(\sim 360 \mathrm{~nm})$ after $65 \mathrm{~min}$ of thermal annealing. Most grains in $\mathrm{MAPbI}_{3}$ films on non-wetting HTLs were much larger than the film thickness, with the average grain size/film thickness aspect ratio reaching $2.3,3.2$ and 7.9 for c-OTPD, PTAA and PCDTBT (Fig. 2f), respectively. 
The largest grain size on PCDTBT reached $\sim 5 \mu \mathrm{m}$, which is about 14-fold of the film thickness; second, most of the grain boundaries are perpendicular to the substrate for films on non-wetting HTLs to minimize the grain boundary energy. It is noted that PCDTBT is so hydrophobic so that the OTP films on PCDTBT were mostly not continuous, and SEM pictures were taken from areas with OTP films.

The influence of surface tension force on the grain nucleation and growth was verified by comparing grain morphology evolution of the $\mathrm{MAPbI}_{3}$ films on two HTLs, wetting PEDOT:PSS and non-wetting c-OTPD. As shown by the SEM images in Fig. 2d,e, the average size of the $\mathrm{MAPbI}_{3}$ grains on c-OTPD $(480 \mathrm{~nm})$ is much larger than that on PEDOT:PSS $(230 \mathrm{~nm})$ just after film drying, verifying that a non-wetting surface suppresses heterogeneous nucleation, results in less dense nuclei and thus larger grain size. The average size of the $\mathrm{MAPbI}_{3}$ grains on c-OTPD increased to $>800 \mathrm{~nm}$ with the largest one reaching $>1.5 \mu \mathrm{m}$ after $65 \mathrm{~min}$ of thermal annealing. In contrast, the average grain size for the $\mathrm{MAPbI}_{3}$ film on PEDOT:PSS remained to be around $300 \mathrm{~nm}$ even after $65 \mathrm{~min}$ of thermal annealing. Finally, the HTLs also impact the crystallinity of the OPT films formed on them. The X-ray diffraction peaks became stronger and sharper for perovskite films on hydrophobic HTLs, agreeing with disappearance of the small grains and better film crystallinity (Fig. 2f,g). No significant change of the diffraction peak ratio was observed, indicating the different HTLs do not cause crystal orientation change.

Influence of the grain aspect ratio on device performance. The influence of the increased grain size and crystallinity on the device performance was evaluated in $\mathrm{PHJ}$ devices with a structure shown in Fig. 3a. Again, c-OPTD and PEDOT:PSS devices were studied because of the excellent reproducibility of the device performance using these two HTLs. Figure $3 \mathrm{~b}$ shows the photocurrent-voltage $(J-V)$ curves of the optimized $\mathrm{MAPbI}_{3}$ devices using c-OTPD and PEDOT:PSS as HTLs. The device with PEDOT:PSS as HTL has a relatively low efficiency of $12.3 \%$, which is consistent with previous result for the devices using only thermal annealing 22 . The device's short circuit current density $\left(J_{\mathrm{SC}}\right)$ increased from 18.6 to $21.2 \mathrm{~mA} \mathrm{~cm}^{-2}$ and open circuit voltage $\left(V_{\mathrm{OC}}\right)$ increased significantly from 0.92 to $1.09 \mathrm{~V}$, when the device's HTL was changed from PEDOT:PSS to c-OTPD. The device employing c-OTPD showed a decent fill factor (FF) of $75.5 \%$, yielding a PCE of $17.5 \%$. Negligible photocurrent hysteresis was observed by changing the scanning directions, as shown in Supplementary Fig. 5. The absence of obvious photocurrent hysteresis is commonly observed in our $\mathrm{PHJ}$ devices because the $\mathrm{MAPbI}_{3}$ films have large grains, and charge traps at the film surface and grain boundaries are well passivated by fullerenes ${ }^{10,12,22,23}$. The devices with thicker $\mathrm{MAPbI}_{3}$ film $(500-600 \mathrm{~nm})$ were fabricated to optimize the PCE. A thicker $\mathrm{MAPbI}_{3}$ active layer at $500 \mathrm{~nm}$ can produce a larger $J_{\mathrm{SC}}$ of $22.4 \mathrm{~mA} \mathrm{~cm}^{-2}$, resulting in an increased PCE of $17.8 \%$, despite of a decrease of $V_{\mathrm{OC}}$ to $1.05 \mathrm{~V}$. Further increasing the film thickness did not increase the $J_{\mathrm{SC}}$ but lowered the FF, which yielded a comparable but slightly lower efficiency of $17.2 \%$, as shown in Fig. 3b. The device performance with varied HTLs, OTPs and OTP film thicknesses is summarized in Table 1. The peak external quantum efficiency (EQE) of the c-OTPD device with 360-nmthick $\mathrm{MAPbI}_{3}$ reached $94.5 \%$ at $390 \mathrm{~nm}$, as shown in Fig. 3c, and the calculated $J_{\mathrm{SC}}$ from EQE for the three devices are in good agreement to measured $J_{\mathrm{SC}}$. The increased $J_{\mathrm{SC}}(21.8$ and $21.5 \mathrm{~mA} \mathrm{~cm}^{-2}$ ) in the device with 500 - and 600-nm-thick $\mathrm{MAPbI}_{3}$ should mostly be ascribed to the improved absorption in the wavelength range of $650-800 \mathrm{~nm}$. The efficiency histogram in Fig. $3 \mathrm{~d}$ shows $>35 \%$ of the devices the PCE higher than $16.0 \%$, and $>80 \%$ devices have PCE higher than $15.0 \%$.

\section{Discussion}

To find the correlation between the device PCE enhancement and larger grain size and better crystallinity, we performed thermal admittance spectroscopy (TAS) measurement to examine the trap density-of-states (tDOS) in the devices with two different HTLs, PEDOT:PSS and c-OTPD. Our previous study showed tDOS of the devices with PEDOT:PSS had a tDOS in the order of $10^{16} \mathrm{~m}^{-3} \mathrm{eV}^{-1}$ in the trap band of $0.310-0.425 \mathrm{eV}$ towards the forbidden gap, which is correlated to grain boundaries ${ }^{4,12}$. As shown in Fig. 3e, the device with c-OTPD HTL had a 10-100-fold smaller tDOS of $10^{14}-10^{15} \mathrm{~m}^{-3} \mathrm{eV}^{-1}$. It should be noticed that this trap density is as low as those measured in single crystals using TAS characterization ${ }^{14}$. The much lower tDOS can be partially explained by significant enlarged $\mathrm{MAPbI}_{3}$ grain size on c-OTPD (Fig. 2b,c). However, it is noted that $\mathrm{MAPbI}_{3}$ grain size on c-OTPD is 2.7-fold that on PEDOT:PSS, which yields only 2.7 times less grain boundary area. If the specific tDOS in unit grain boundary area is the same for the films on both HTLs, there should be additional factors contributed the dramatically reduced tDOS. We speculate that the improved quality of $\mathrm{c}-\mathrm{OTPD} / \mathrm{MAPbI}_{3}$ interface also contributes to the tDOS reduction. This speculation is supported by the observed large peak EQE of $94.5 \%$ at the $390 \mathrm{~nm}$ in the device with c-OTPD, which is much higher than the devices with PEDOT:PSS ${ }^{10,12,13}$. Since shorter-wavelength light has shorter penetration depth in OTPs, the generated charges are more susceptible to charge recombination at the $\mathrm{HTL} / \mathrm{MAPbI}_{3}$ interface. A high EQE above $90 \%$ at shorter wavelength proves the very good passivation of charge traps in $\mathrm{MAPbI}_{3}$ bottom surface close to the transparent electrode side.

The reduction of $\mathrm{tDOS}$ at $\mathrm{MAPbI}_{3}$ bottom surface (c-OTPD side) was further confirmed by photoluminescence study. In our previous study of passivation of $\mathrm{MAPbI}_{3}$ top surface by fullerenes, a blue shift of photoluminescence was observed for the fullerene-passivated $\mathrm{MAPbI}_{3}$ when exciting light was from the top side, while there was no photoluminescence blue shift if the excitation light was from the bottom side, because fullerenes cannot reach the bottom surface of $\mathrm{MAPbI}_{3}$ even after $1 \mathrm{~h}$ thermal annealing. Here we observed a blue shift of photoluminescence peak from 784 to $774 \mathrm{~nm}$ for the c-OTPD/ $\mathrm{MAPbI}_{3}$ films with incident light from indium tin oxide (ITO) side, as shown in Fig. 3f, confirming the scenario ${ }^{4,12}$. The absence of charge traps at $\mathrm{MAPbI}_{3}$ bottom surface can be explained by (1) the absence of small $\mathrm{MAPbI}_{3}$ grains close to c-OTPD and (2) excellent crystallinity and stoichiometry of the bottom surface. The large surface energy-driven interaction pushes any residual ions to merge to large grains, resembling the hydrophobic interaction that was used for single-crystal nanoparticle synthesis ${ }^{24}$. We speculate the vertical and straight grain boundaries facilitate the diffusion of fullerenes, allowing them move towards the bottom surface, which reduced the trap density near the bottom side.

Now that both top and bottom surfaces of the $\mathrm{MAPbI}_{3}$ thin films have low trap density, which resembles the passivation of silicon wafer at all surfaces by oxidization, the charge recombination lifetime $\left(\tau_{\mathrm{r}}\right)$ should be significantly elongated. As shown in Fig. $3 g$, the $\tau_{\mathrm{r}}$ measured by impedance spectroscopy is significantly prolonged by 7-10 times in the bias region of $0-0.7 \mathrm{~V}$ after replacing PEDOT:PSS with c-OTPD. $\tau_{\mathrm{r}}$ in c-OTPD device at $0 \mathrm{~V}$ reached $69 \mu \mathrm{s}$, which is close to that of $\mathrm{MAPbI}_{3}$ single crystals ${ }^{14}$. The carrier diffusion length in these large crystalline grains should be comparable to that in $\mathrm{MAPbI}_{3}$ single crystals, which was demonstrated to exceed $175 \mu \mathrm{m}$ under 1 sun 

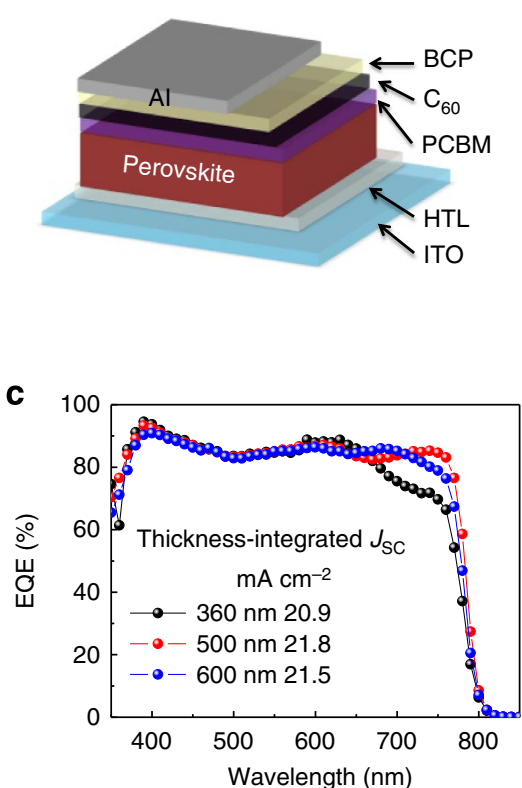

e

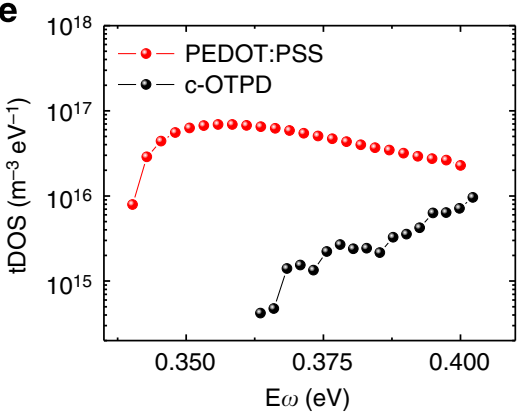

g

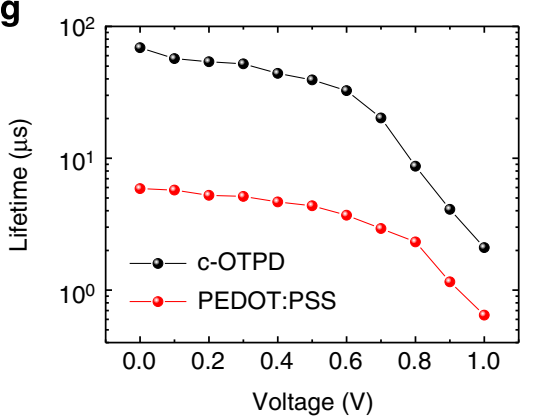

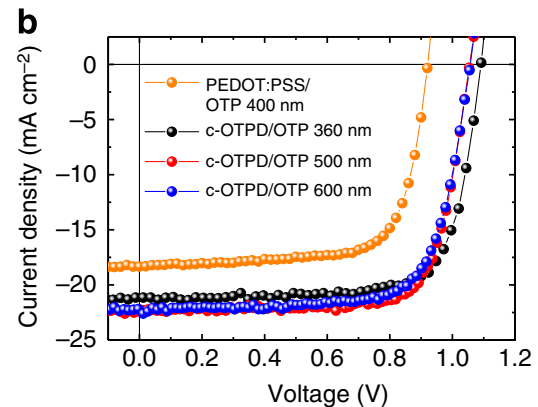

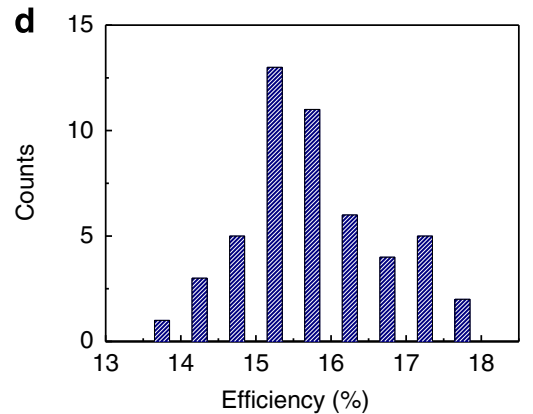

f

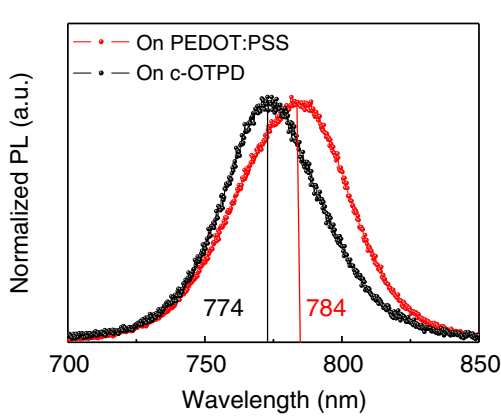

h

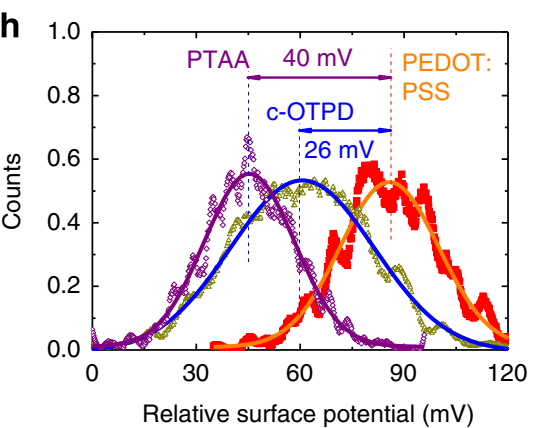

Figure 3 | Characterization of the MAPbl $_{3}$ devices with PEDOT:PSS and c-OTPD HTLs. (a) The PHJ device structure; (b) J- $V$ of the devices with PEDOT:PSS and c-OTPD HTLs, and with different MAPbl $\left.\right|_{3}$ thickness of 360, 500 and $600 \mathrm{~nm}$; (c) EQE of the devices with c-OTPD HTL and with different $\mathrm{MAPb}_{3}$ thickness of 360,500 and $600 \mathrm{~nm}$; (d) efficiency histogram of the 50 devices using c-OTPD as HTL; (e) tdOS, (f) photoluminescence at room temperature and (g) impedance spectroscopy lifetime at different bias for the devices with PEDOT:PSS and c-OTPD HTLs. (h) Work function distribution of the HTLS of PEDOT:PSS, c-OTPD and PTAA.

Table 1 | Summary of the best device performance for the OTP devices with different HTLs and different thickness of active layers.

\begin{tabular}{|c|c|c|c|c|}
\hline $\begin{array}{l}\text { HTL and active layer } \\
\text { thickness }\end{array}$ & $\begin{array}{c}J_{s c} \\
(m A ~ c m\end{array}$ & $\begin{array}{l}V_{\text {oc }} \\
(V)\end{array}$ & $\begin{array}{c}\mathbf{F F} \\
(\%)\end{array}$ & $\begin{array}{l}\text { PCE } \\
(\%)\end{array}$ \\
\hline PEDOT:PSS $400 \mathrm{~nm} \mathrm{MAPbl}_{3}$ & 18.6 & 0.92 & 72.0 & 12.3 \\
\hline c-OTPD $360 \mathrm{~nm} \mathrm{MAPb|_{3 }}$ & 21.2 & 1.09 & 75.5 & 17.5 \\
\hline c-OTPD $500 \mathrm{~nm} \mathrm{MAPbl}_{3}$ & 22.4 & 1.05 & 75.6 & 17.8 \\
\hline $\mathrm{c}-\mathrm{OTPD} 600 \mathrm{~nm} \mathrm{MAPbl_{3 }}$ & 22.2 & 1.06 & 73.4 & 17.2 \\
\hline PTAA $500 \mathrm{~nm} \mathrm{MAPbl}{ }_{3}$ & 22.0 & 1.07 & 76.8 & 18.1 \\
\hline
\end{tabular}

illumination because of the same carrier recombination lifetime and mobility 4,14 , which is already more than 100 times longer than the film's thickness. Therefore, all the photogenerated charges can diffuse to the charge transport layers or grain boundaries without recombination, and the device efficiency is only determined by the charge recombination at grain boundaries or at electrode interfaces. It clearly demonstrated that excellent capability of non-wetting c-OTPD HTL in reducing trap density and suppressing charge recombination in $\mathrm{MAPbI}_{3}$. A longer $\tau_{\mathrm{r}}$ is expected to contribute to the observed increase of $J_{\mathrm{SC}}, V_{\mathrm{OC}}$ and $\mathrm{FF}$, while partial of the increased $V_{\mathrm{OC}}$ should be ascribed to a higher work function of c-OTPD than PEDOT:PSS. The work 
function of different HTLs was directly compared by Kelvin probe force microscopy measurement. Gold films deposited on both c-OTPD and PEDOT:PSS surface in the same batch were used as work function reference. As shown in Fig. $3 \mathrm{~h}$ and Supplementary Fig. 6, a higher work function by $25 \mathrm{meV}$ for c-OTPD than PEDOT:PSS can be derived. The work function difference of the HTLs is much smaller than $V_{\mathrm{OC}}$ improvement, indicating that the contribution from the reduced charge recombination due to the larger and better OTP grains formed on the non-wetting surface dominates the $V_{\mathrm{OC}}$ enhancement observed here. This conclusion is further supported by the a recent observation by Kim and coworkers ${ }^{25}$ that a HTL with work function of $5.4 \mathrm{eV}$, which is higher than ours, was applied in a same device structure with ours, while the highest $V_{\mathrm{OC}}$ was only $0.98 \mathrm{~V}$. The much lower $V_{\mathrm{OC}}$ in that work can be explained by the very small grain sizes.

It is noted that PTAA has larger OTP grains on it and a slightly higher work function than C-OTPD, therefore we finally optimized our device performance using PTAA HTL. Although larger OTP grains were observed on PCDTBT, most OTP films on PCDTBT have low film coverage $<100 \%$, as shown in Supplementary Fig. 7, and thus not suitable for device optimization. The PTAA layer was doped by $1 \mathrm{wt} \%$ tetrafluoro-tetracyanoquinodimethane (F4-TCNQ) to enhance its conductivity. Here $\mathrm{MAPbI}_{3}$ with a thickness of $500 \mathrm{~nm}$ by the two-step interdiffusion method was fabricated. The optimized device performances are shown in Fig. $4 \mathrm{a}$, and are summarized in Table 1. Compared with the device with c-OTPD, the $\mathrm{MAPbI}_{3}$ device with PTAA showed a slightly higher efficiency of $18.1 \%$ with comparable $V_{\mathrm{OC}}$ of $1.07 \mathrm{~V}$ and $J_{\mathrm{SC}}$ of $22.0 \mathrm{~mA} \mathrm{~cm}^{-2}$ but a larger $\mathrm{FF}$ of $76.8 \%$. The calculated $J_{\mathrm{SC}}$ from EQE in Fig. $4 \mathrm{~b}$ reached $21.6 \mathrm{~mA} \mathrm{~cm}^{-2}$, which is in good agreement with measured photocurrent. The photocurrent measured at $0.88 \mathrm{~V}$ slightly increased from initial 20.5 to $20.8 \mathrm{~mA} \mathrm{~cm}^{-2}$ (stabilized) after 60-s illumination, resulting in an increased stabilized PCE of $18.3 \%$ for the device with PTAA HTL (Fig. 4c). The quick turning on the photocurrent confirms the absence of large density charge traps, while the slow climbing of the $J_{\mathrm{SC}}$ to the maximum value indicates the presence a relatively small density of deep traps in the devices despite of the passivation of traps on perovskite surface and grain boundaries by the double fullerene layer, which might originate from the deep traps in the fullerene layer itself.

In summary, the non-wetting HTLs were demonstrated to be effective in enhancing the efficiency of OTP PHJ devices to $18.3 \%$. In addition to the higher PCE, these HTLs are expected to be much more stable than the acidic PEDOT:PSS. This work provided a simple method of achieving high-aspect, low-defect density, high-quality perovskite polycrystalline thin films, which possess much better optoelectronic properties such as fewer bulk and surface traps, and higher carrier mobilities. The improved property can potentially give rise to the applications in other fields, such as high mobility for transistors, higher responsivity and lower pink noise for photodetectors by removing the charge traps $^{26}$, lower driving voltage for light emitting diodes and lower threshold excitation density and higher efficiency for lasers.

\section{Methods}

HTL and OTP layer fabrication. To prepare the OTPD solution for spin coating, diphenyliodonium-hexafluorophosphate was added into OTPD solution with a weight ratio of $2-5 \mathrm{wt} \%$ to OTPD as photoinitiator. For c-OTPD film fabrication, 0.25 wt\% OTPD solution was first spun on the ITO substrate at 6,000 r.p.m. for $35 \mathrm{~s}$, and then the as-prepared OTPD film was crosslinked by ultraviolet curing (wavelength of $365 \mathrm{~nm}$ ) for $3 \mathrm{~min}$. To promote the crosslinking, the ultraviolet cured c-OTPD film was thermally annealed at $110^{\circ} \mathrm{C}$ for $10 \mathrm{~min}$. PTAA film was prepared by spin coating $0.5 \mathrm{wt} \%$ PTAA solution doped with $1 \mathrm{wt} \%$ F4-TCNQ at 6,000 r.p.m., and the as-prepared film thermally annealed at $110^{\circ} \mathrm{C}$ for $10 \mathrm{~min}$.
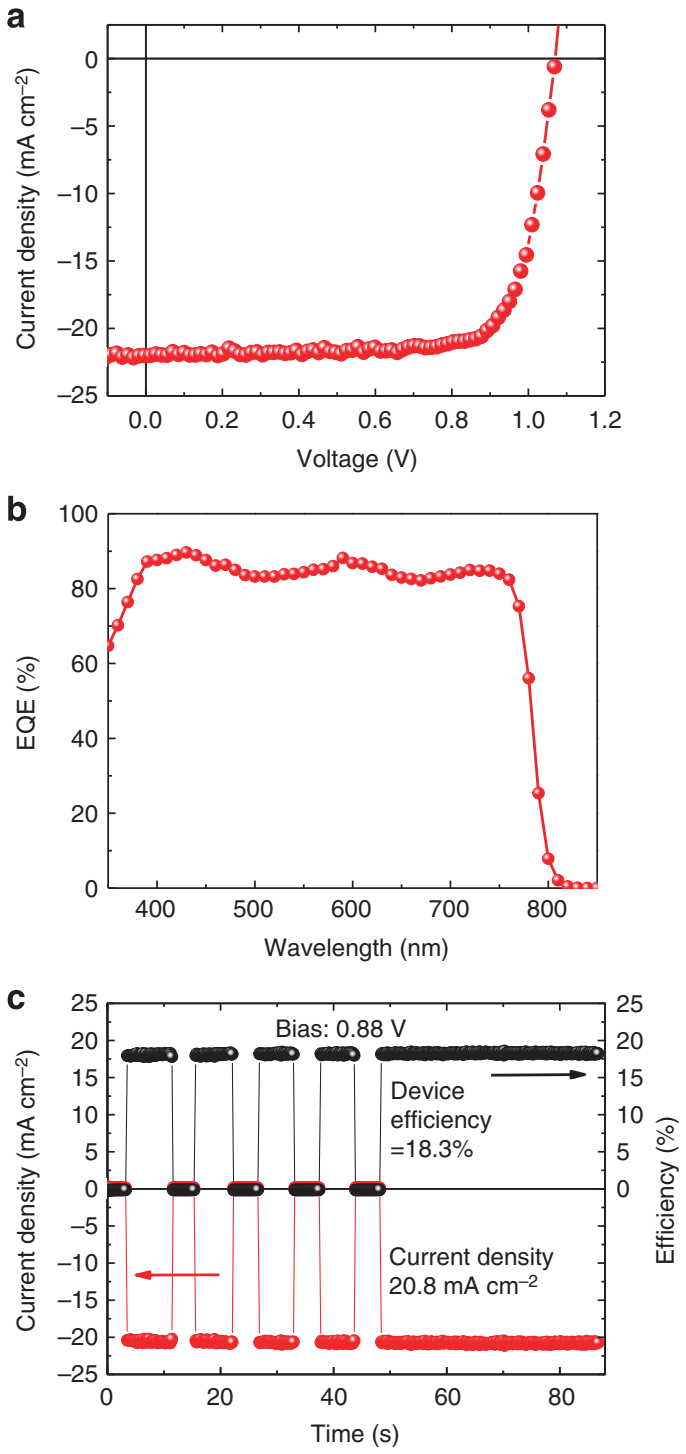

Figure 4 | Performance of the MAPbl $_{3}$ devices with PTAA HTL. (a) The photocurrent of the $\mathrm{MAPbl}_{3}$ devices under 1 sun illumination; (b) EQE of the devices with the active layers of $\mathrm{MAPb}_{3} ;$ (c) stabilized photocurrent measurement of the $\mathrm{MAPb}_{3}$ device with PTAA HTL under 1 sun illumination with light turned on and off by a shutter.

PCDTBT was prepared by spin coating $0.5 \mathrm{wt} \%$ PCDTBT solution at 6,000 r.p.m. and the as-prepared film thermally annealed at $110^{\circ} \mathrm{C}$ for $10 \mathrm{~min}$. PEDOT:PSS (Baytron-P 4083) and $1 \mathrm{wt} \%$ PVA solution was spin coated on clean ITO substrate at a speed of 3,000 r.p.m. The films were then annealed at $130^{\circ} \mathrm{C}$ for $30 \mathrm{~min}$.

The $\mathrm{MAPbI}_{3}$ films were fabricated by thermal annealing-induced interdiffusion method ${ }^{10}$. The $\mathrm{PbI}_{2}$ layers on all HTLs were spin coated from $110^{\circ} \mathrm{C}$ pre-heated $\mathrm{PbI}_{2}$ solution in DMF. $\mathrm{PbI}_{2}$ beads $(99.999 \%$ trace metals basis) were purchased from Sigma-Aldrich, which have good solubility in DMF. Around $60 \mu \mathrm{l}$ of hot $\mathrm{PbI}_{2}$ precursor solution, which was pre-heated to $110^{\circ} \mathrm{C}$, was directly transferred by plastic (polypropylene) disposable pipettes from the bottle of heated $\mathrm{PbI}_{2}$ solution to the HTL-covered ITO substrates within $2 \mathrm{~s}$. The spinning was quickly started at the speed of 6,000 r.p.m. after the injection of $\mathrm{PbI}_{2}$ solution. The as-fabricated $\mathrm{PbI}_{2}$ films were dried and annealed at $110^{\circ} \mathrm{C}$ for $5 \mathrm{~min}$. To fabricate 360 -, 500- and 600-nm-thick $\mathrm{MAPbI}_{3}$ film, 600, 700 and $800 \mathrm{mg} \mathrm{ml}^{-1} \mathrm{PbI}_{2}$ DMF precursor solutions were used with 70,80 and $95 \mathrm{mg} \mathrm{ml}^{-1}$ methylammonium iodide (MAI) 2-propanol precursor solution, respectively. The MAI was synthesized from methylamine ( $40 \mathrm{wt} \%$ in $\mathrm{H}_{2} \mathrm{O}$, Sigma-Aldrich) and hydroiodic acid ( $57 \mathrm{wt} \%$ in $\mathrm{H}_{2} \mathrm{O}, 99.95 \%$, with stabilizer, Sigma-Aldrich) and the method was reported elsewhere ${ }^{9}$. The deposition of MAI layer was taken at 6,000 r.p.m. and from a hot precursor solution at $70^{\circ} \mathrm{C}$. The stacked precursor layers were annealed on the hotplate at $105^{\circ} \mathrm{C}$ with a Petri dish covering them. The following deposition of phenyl-C61-butyric acid methyl ester, $C_{60}, 2,9$-dimethyl-4,7-diphenyl-1,10- 
phenanthroline and $\mathrm{Al}$ layers were reported elsewhere ${ }^{10,12,19}$. The device working area was $7.25 \mathrm{~mm}^{2}$, defined by the overlap of ITO substrate and $\mathrm{Al}$ cathode.

Film and device characterization. The microscope images of $\mathrm{PbI}_{2}$ layer spin coated at 70 and $110^{\circ} \mathrm{C}$ were taken by an optical microscope Olympus BX61, with an integrated charge-coupled device (Photometrics, CoolSNAP-cf). X-ray diffraction pattern was obtained by a Rigaku D/Max-B X-ray diffractometer with Bragg-Brentano parafocusing geometry. A Co-K $\alpha$ tube was equipped in the diffractometer with an emitting wavelength of $1.79 \AA$. The SEM images were taken from a Quanta 200 FEG environmental scanning electron microscope. The grain size was obtained by measuring the average diameter of the grains in the plane direction from SEM images. A Xenon lamp-based solar simulator (Oriel 67005, $150 \mathrm{~W}$ solar simulator) was used to produce the simulated AM 1.5G irradiation $\left(100 \mathrm{~mW} \mathrm{~cm}^{-2}\right)$, and the calibration of the light was carried out by a Si diode (Hamamatsu S1133) equipped with a Schott visible-colour glass filter (KG5 colour filter). The bias scanning rate was $0.13 \mathrm{~V} \mathrm{~s}^{-1}$ for the device $J-V$ curve measurement. TAS measurement was performed by a LCR (inductance (L), capacitance (C), and resistance (R)) meter (Agilent E4980A) to obtain the devices' frequency-dependent capacitance and voltage-dependent capacitance, which was used for devices' tDOS derivation. The derivation procedure was reported elsewhere ${ }^{4}$. Impedance spectroscopy was also recorded by the LCR meter (Agilent E4980A) with home-made software. The devices were kept under 1 sun illumination at room temperature during the measurement. The recombination lifetime equates to the reciprocal of the angular frequency at the top of the arc in impedance spectra with the Nyquist plot $^{27}$ (Supplementary Fig. 8), which has good agreement with the fitted results from recombination resistance and chemical capacitance (Supplementary Figs 9 and 10).

\section{References}

1. Burschka, J. et al. Sequential deposition as a route to high-performance perovskite-sensitized solar cells. Nature 499, 316-319 (2013).

2. Liu, M., Johnston, M. B. \& Snaith, H. J. Efficient planar heterojunction perovskite solar cells by vapour deposition. Nature 501, 395-398 (2013).

3. Im, J.-H. et al. Growth of $\mathrm{CH}_{3} \mathrm{NH}_{3} \mathrm{PbI}_{3}$ cuboids with controlled size for high-efficiency perovskite solar cells. Nat. Nanotechnol. 9, 927-932 (2014).

4. Shao, Y. et al. Origin and elimination of photocurrent hysteresis by fullerene passivation in $\mathrm{CH}_{3} \mathrm{NH}_{3} \mathrm{PbI}_{3}$ planar heterojunction solar cells. Nat. Commun. 5, 5784 (2014)

5. Xiao, Z. et al. Giant switchable photovoltaic effect in organometal trihalide perovskite devices. Nat. Mater. 14, 193-198 (2014).

6. Green, M. A., Ho-Baillie, A. \& Snaith, H. J. The emergence of perovskite solar cells. Nat. Photon. 8, 506-514 (2014).

7. Malinkiewicz, O. et al. Perovskite solar cells employing organic chargetransport layers. Nat. Photon. 8, 128-132 (2014).

8. Zhou, H. et al. Interface engineering of highly efficient perovskite solar cells. Science 345, 542-546 (2014)

9. Lee, M. M. et al. Efficient hybrid solar cells based on meso-superstructured organometal halide perovskites. Science 338, 643-647 (2012)

10. Xiao, Z. et al. Efficient, high yield perovskite photovoltaic devices grown by interdiffusion of solution-processed precursor stacking layers. Energy Environ. Sci. 7, 2619-2623 (2014)

11. Kojima, A., Teshima, K., Shirai, Y. \& Miyasaka, T. Organometal halide perovskites as visible-light sensitizers for photovoltaic cells. J. Am. Chem. Soc. 131, 6050-6051 (2009).

12. Xiao, Z. et al. Solvent-annealing of perovskite induced crystal growth for photovoltaic device efficiency enhancement. Adv. Mater. 26, 6503-6509 (2014).

13. Chen, Q. et al. Planar heterojunction perovskite solar cells via vapor-assisted solution process. J. Am. Chem. Soc. 136, 622-625 (2013).

14. Dong, Q. et al. Electron-hole diffusion length exceeding 3 millimeters in low-temperature solution grown $\mathrm{CH}_{3} \mathrm{NH}_{3} \mathrm{PbI}_{3}$ single crystals. Science 27, 967-970 (2015)

15. Zhao, D. et al. High-efficiency solution-processed planar perovskite solar cells with a polymer hole transport layer. Adv. Energy Mater. 5, 1401855 (2015).

16. Eperon, G. E. et al. Morphological control for high performance, solutionprocessed planar heterojunction perovskite solar cells. Adv. Funct. Mater. 24, 151-157 (2014)
17. Conings, B. et al. Perovskite-based hybrid solar cells exceeding $10 \%$ efficiency with high reproducibility using a thin film sandwich approach. Adv. Mater. 26, 2041-2046 (2014).

18. Wang, L. et al. Femtosecond time-resolved transient absorption spectroscopy of $\mathrm{CH}_{3} \mathrm{NH}_{3} \mathrm{PbI}_{3}$ perovskite films: evidence for passivation effect of $\mathrm{PbI}_{2}$. J. Am. Chem. Soc. 136, 12205-12208 (2014).

19. Chen, Q. et al. Controllable self-induced passivation of hybrid lead iodide perovskites toward high performance solar cells. Nano Lett. 14, 4158-4163 (2014).

20. Noel, N. K. et al. Enhanced photoluminescence and solar cell performance via lewis base passivation of organic-inorganic lead halide perovskites. ACS Nano 8, 9815-9821 (2014).

21. Jeon, N. J. et al. Solvent engineering for high-performance inorganic-organic hybrid perovskite solar cells. Nat. Mater. 13, 897-903 (2014).

22. Bi, C. et al. Understanding the formation and evolution of interdiffusion grown organolead halide perovskite thin films by thermal annealing. J. Mater. Chem. A 2, 18508-18514 (2014).

23. Wang, Q. et al. Large fill-factor bilayer iodine perovskite solar cells fabricated by a low-temperature solution-process. Energy Environ. Sci. 7, 2359-2365 (2014).

24. Xiao, Z. et al. Synthesis and application of ferroelectric P(VDF-TrFE) nanoparticles in organic photovoltaic devices for high efficiency. Adv. Energy Mater. 3, 1581-1588 (2013).

25. Lim, K.-G. et al. Boosting the power conversion efficiency of perovskite solar cells using self-organized polymeric hole extraction layers with high work function. Adv. Mater. 26, 6461-6466 (2014).

26. Fang, Y. et al. Resolving weak light of sub-picowatt per square centimeter by hybrid perovskite photodetectors enabled by noise reduction. Adv. Mater. 27, 2804-2810 (2015).

27. Mora-Seró, I. et al. Impedance spectroscopy characterisation of highly efficient silicon solar cells under different light illumination intensities. Energy Environ. Sci. 2, 678-686 (2009).

\section{Acknowledgements}

We thank the financial support from Department of Energy under Award DE-EE0006709, National Science Foundation under Awards ECCS-1201384 and ECCS-1252623 and the Nebraska Public Power District through the Nebraska Center for Energy Sciences Research.

\section{Author contributions}

J.H. conceived the idea; C.B. conducted most of device fabrication and device measurement, SEM and photoluminescence; Y.S. measured trap density, carrier lifetime and surface work function; Y.Y. conducted the X-ray diffraction measurement; J.H. wrote the paper. Q.W contributed to the contact angle measurement; Z.X. provided the Supplementary Fig. 1.

\section{Additional information}

Supplementary Information accompanies this paper at http://www.nature.com/ naturecommunications

Competing financial interests: The authors declare no competing financial interests.

Reprints and permission information is available online at http://npg.nature.com/ reprintsandpermissions/

How to cite this article: $\mathrm{Bi}, \mathrm{C}$. et al. Non-wetting surface-driven high-aspect-ratio crystalline grain growth for efficient hybrid perovskite solar cells. Nat. Commun. 6:7747 doi: 10.1038/ncomms8747 (2015)

This work is licensed under a Creative Commons Attribution 4.0 International License. The images or other third party material in this article are included in the article's Creative Commons license, unless indicated otherwise in the credit line; if the material is not included under the Creative Commons license, users will need to obtain permission from the license holder to reproduce the material To view a copy of this license, visit http://creativecommons.org/licenses/by/4.0/ 